\title{
ON THE REDUCTION OF THE IDENTIFICATION OF THE PARAMETERS OF A DIFFERENTIAL EQUATION IN A HILBERT SPACE TO A BOUNDARY VALUE PROBLEM
}

\author{
O. NAKONECHNYI AND V. MARTSENYUK
}

Received 17 January, 2019

\begin{abstract}
The work considers the problem of differential equation parameter identification given in Hilbert space. Conditions of existence of problem solutions are established. They correspond to conditions of continuous dependence of solutions of differential equations on parameters. In case of linear model and space $L_{2}$ condition means convergence in mean quadratic sense on aprioric set.

A constructive algorithm of identification problem solution in Hilbert space is developed. It is based on the considering of the estimation of differential equation solution and it results in the solving corresponding boundary value problem. The way of its reduction to initial value problems is offered. One partial case allowing problem solution not only in operator form is considered.
\end{abstract}

2010 Mathematics Subject Classification: 34G99; 49N99; 34K10

Keywords: differential equations, identification problem, Hilbert space, boundary value problem, Riccati equation

\section{INTRODUCTION}

When modeling physical and biological processes of real world [9], [10], [11], [12], [14], [15] problems of search of system parameter estimates which can be elements of some functional spaces arise. Such models include differential equations with delay, integro-differential equations. Their theory is presented in works [5], [6]. Some results on parameter identification of these systems were presented in earlier monographs [2], [13]. Infinite-dimensional minimax problems were displayed in [8], [17].

Last time there appeared interest on such problems dealing with investigation of the "solution semigroup and the structural operators in the initial data space" [3], [7]. As a rule the results are presented "in terms of the initial values and eigenvectors of adjoint operator" [7]. Practical applications require more constructive presentations of the methods in the form of initial or boundary value problems. 
Hence, the purpose of this research is to establish conditions of solution existence for parameter identification problems given in abstract Hilbert spaces and development of constructive algorithms resulting in corresponding boundary value problems.

\section{CONDITIONS FOR EXISTENCE OF SOLUTIONS OF IDENTIFICATION PROBLEMS IN HILBERT SPACE}

In order to solve identification problems in Hilbert space we need to establish some conditions of existence of solutions of these problems. The conditions that will be presented are based on the following theoretical background.

Let $\mathbb{W}$ be abstract Hilbert space of parameters $\alpha$ which should be identified. Aprioric set of parameters $G$ is often described as

$$
G=\{\alpha \in \mathbb{W}: \mathcal{g}(\alpha) \leq 1\}
$$

where $\mathcal{f}(\alpha), \alpha \in \mathbb{W}$ is some functional. It is natural to choose as aposterioric estimate for $\alpha \in \mathbb{W}$ (denote it as $\hat{\alpha}$ ) minimum point of functional $J(\alpha)$, that is

$$
\hat{\alpha}=\arg \min _{\alpha \in G} \mathcal{g}(\alpha) .
$$

In case of quadratic functional $\mathcal{I}(\alpha)$ such an estimate corresponds to Chebyshev's center.

Thus, firstly let's establish existence conditions for minimum point of functional $\mathcal{L}(\alpha)$.

Definition 1. Functional $\mathcal{g}(\alpha), \alpha \in \mathbb{W}$ is said to be weakly lower semicontinuous at point $\alpha_{0}$ if for arbitrary sequence $\left\{\alpha_{n}\right\}_{n=1, \infty}$ which converges weakly to $\alpha_{0}$, that is $\forall \beta \in \mathbb{W} \quad\left(\alpha_{n}, \beta\right)_{\mathbb{W}} \underset{n \rightarrow \infty}{\rightarrow}\left(\alpha_{0}, \beta\right)_{\mathbb{W}}$ holds

$$
\varliminf_{n \rightarrow \infty} \mathcal{f}\left(\alpha_{n}\right) \geq \mathcal{f}\left(\alpha_{0}\right)
$$

The following result follows from the generalized Weierstrass Theorem ([1], Theorem 7.3.1)

Theorem 1. Let

(i) $G \subset \mathbb{Q}$ be bounded and weakly closed set;

(ii) $\mathcal{g}: \mathbb{H} \rightarrow R$ is weakly lower semicontinuous.

Then there is point $\alpha_{0} \in G$ such that

$$
\min _{\alpha \in G} \mathcal{f}(\alpha)=\mathcal{H}\left(\alpha_{0}\right)
$$

Solving identification problems in Hilbert spaces they consider restrictions given by functionals of the following form

$$
\mathcal{g}(\alpha)=\int_{0}^{T}(Q(y(t)-H x(t)),(y(t)-H x(t))) d t=\mathscr{g}_{1}(x)
$$


where

$$
Q>0
$$

is some given positive definite matrix, $x(t) \in \mathbb{R}^{n}$ is system state dependent on parameter $\alpha \in \mathbb{W}$. Note, that as it follows from (2.3) functional (2.2) is lower continuous with respect to $x \in \mathbb{R}^{n}$. It is left to show that functional (2.2) is weakly lower semicontinuous with respect to $\alpha \in G$.

So, in order to prove existence of minimum point of functional $\mathcal{f}(\alpha)$ of the form (2.2) (in other words, existence of aposterioric estimate) it is necessary to establish conditions of continuous dependence $x(t)$ with respect to $\alpha$.

Consider the following system

$$
\left\{\begin{aligned}
\frac{d x(t)}{d t} & =\mathcal{A}(t, \alpha) x(t) \\
x\left(t_{0}\right) & =x_{0}
\end{aligned}\right.
$$

where $x(t) \in \mathbb{C}^{1}\left([0, T], \mathbb{R}^{n}\right), \mathcal{A}(t, \alpha):[0, T] \times G \times \mathbb{C}^{1}\left([0, T], \mathbb{R}^{n}\right) \rightarrow \mathbb{C}\left([0, T], \mathbb{R}^{n}\right)$ is linear operator with respect to $x(t)$.

Lemma 1. Let operator $\mathcal{A}(\bullet, \bullet)$ be linear with respect to $x(t)$ and is such that

(i) $\|\mathcal{A}(s, \alpha)\| \leq k(s)$ at $\alpha \in G, s \in\left[t_{0}, T\right]$;

(ii) for arbitrary $x(s) \in \mathbb{C}^{1}\left([0, T], \mathbb{R}^{n}\right)$, arbitrary $\alpha_{0} \in G$ and $\alpha_{n} \underset{n \rightarrow \infty}{\rightarrow} \alpha_{0}$ it holds

$$
\lim _{\alpha_{n} \rightarrow \alpha_{0}} \int_{t^{\prime}}^{t^{\prime \prime}}\left[\mathcal{A}\left(s, \alpha_{n}\right) x(s)-\mathcal{A}\left(s, \alpha_{0}\right) x(s)\right] d s=0
$$

Then solution (2.4) $x(t)$ depends on $\alpha \in G$ continuously.

Proof. Let's denote solution (2.4) corresponding to parameter $\alpha \in \mathbb{W}$ as $x(t, \alpha)$. Further we estimate

$$
\begin{aligned}
& \left\|x\left(t, \alpha_{n}\right)-x\left(t, \alpha_{0}\right)\right\|= \\
& =\left\|x\left(t_{0}, \alpha_{n}\right)+\int_{t_{0}}^{t} \mathcal{A}\left(s, \alpha_{n}\right) x\left(s, \alpha_{n}\right) d s-x\left(t_{0}, \alpha_{0}\right)-\int_{t_{0}}^{t} \mathcal{A}\left(s, \alpha_{0}\right) x\left(s, \alpha_{0}\right) d s\right\| \leq \\
& \quad \leq\left\|x\left(t_{0}, \alpha_{n}\right)-x\left(t_{0}, \alpha_{0}\right)\right\|+\left\|\int_{t_{0}}^{t} \mathcal{A}\left(s, \alpha_{n}\right) x\left(s, \alpha_{n}\right) d s-\int_{t_{0}}^{t} \mathcal{A}\left(s, \alpha_{0}\right) x\left(s, \alpha_{0}\right) d s\right\| \leq \\
& \quad \leq\left\|x\left(t_{0}, \alpha_{n}\right)-x\left(t_{0}, \alpha_{0}\right)\right\|+\left\|\int_{t_{0}}^{t} \mathcal{A}\left(s, \alpha_{n}\right) x\left(s, \alpha_{0}\right) d s-\int_{t_{0}}^{t} \mathcal{A}\left(s, \alpha_{0}\right) x\left(s, \alpha_{0}\right) d s\right\|+ \\
& \quad+\left\|\int_{t_{0}}^{t} \mathcal{A}\left(s, \alpha_{n}\right) x\left(s, \alpha_{n}\right) d s-\int_{t_{0}}^{t} \mathcal{A}\left(s, \alpha_{n}\right) x\left(s, \alpha_{0}\right) d s\right\| \leq \int_{t_{0}}^{t} \mathcal{A}\left(s, \alpha_{0}\right) x\left(s, \alpha_{0}\right) d s \|+ \\
& \quad \leq\left\|x\left(t_{0}, \alpha_{n}\right)-x\left(t_{0}, \alpha_{0}\right)\right\|+\| \int_{t_{0}}^{t} \mathcal{A}\left(s, \alpha_{n}\right) x\left(s, \alpha_{0}\right) d s-\int^{t} \\
& \quad+\int_{t_{0}}^{t} k(s)\left\|x\left(s, \alpha_{n}\right)-x\left(s, \alpha_{0}\right)\right\| d s
\end{aligned}
$$


Due to Gronwall lemma

$$
\begin{aligned}
& \left\|x\left(t, \alpha_{n}\right)-x\left(t, \alpha_{0}\right)\right\| \leq \\
& \leq\left(\left\|x\left(t_{0}, \alpha_{n}\right)-x\left(t_{0}, \alpha_{0}\right)\right\|+\left\|\int_{t_{0}}^{t} \mathcal{A}\left(s, \alpha_{n}\right) x\left(s, \alpha_{0}\right) d s-\int_{t_{0}}^{t} \mathcal{A}\left(s, \alpha_{0}\right) x\left(s, \alpha_{0}\right) d s\right\|\right) \\
& \quad e^{\left\|\int_{t_{0}}^{t} k(s) d s\right\|}
\end{aligned}
$$

Taking into account (2.5) right side of inequality (2.6) tends to zero as $\alpha_{n} \underset{n \rightarrow \infty}{\rightarrow} \alpha_{0}$.

Corollary 1. If operator $\mathcal{A}(s, \alpha)$ is linear with respect to $\alpha$ then condition (2.5) can be rewritten as

$$
\lim _{\alpha_{n} \rightarrow \alpha_{0}} \int_{t^{\prime}}^{t^{\prime \prime}}\left\|\mathcal{A}\left(s, \alpha_{n}\right)-\mathcal{A}\left(s, \alpha_{0}\right)\right\| d s=0
$$

Corollary 2. If operator $\mathcal{A}(s, \alpha)$ is linear with respect to $\alpha$ and doesn't depend on $t$, that is $\mathcal{A}(s, \alpha)=\mathcal{A}(\alpha)$ then condition (2.5) can be rewritten as

$$
\lim _{\alpha_{n} \rightarrow \alpha_{0}}\left\|\mathcal{A}\left(\alpha_{n}\right)-\mathcal{A}\left(\alpha_{0}\right)\right\|=0
$$

\section{LINEAR SYSTEM WITH INTEGRAL KERNEL}

Consider the following system which is partial case of (2.4)

$$
\left\{\begin{array}{l}
\frac{d x(t)}{d t}=A x(t)+\int_{0}^{t} K(t-s) x(s) d s \\
x(0)=x_{0}
\end{array}\right.
$$

where $A, K(s) \in \mathbb{R}^{n \times n}$ are matrix and matrix-valued function correspondingly.

Lemma 2. Let matrix-valued function $K(s)$ belong to set $G$ which satisfies to (i) for any $K_{0}(s) \in G$ and $K_{n} \underset{n \rightarrow \infty}{\rightarrow} K_{0}$ we have

$$
\lim _{n \rightarrow \infty} \int_{0}^{T}\left\|K_{n}(\tau)-K_{0}(\tau)\right\|^{2} d \tau=0
$$

That is we require convergence in strong-quadratic sense.

Then the solution (2.4) $x(t)$ depends on $K(\bullet) \in G$ continuously. 
Proof. Denote solution of (3.1) corresponding to some integral kernel $K(\bullet)$ as $x(t, K)$. Let's estimate

$$
\begin{aligned}
& \left\|x\left(t, K_{n}\right)-x\left(t, K_{0}\right)\right\|= \\
& =\left\|\begin{array}{l}
x\left(0, K_{n}\right)+\int_{0}^{t} \int_{0}^{t} K_{n}\left(t_{1}-s\right) x\left(s, K_{n}\right) d s d t_{1}-x\left(0, K_{0}\right) \\
-\int_{0}^{t} \int_{0}^{t} K_{0}\left(t_{1}-s\right) x\left(s, K_{0}\right) d s d t_{1}+\int_{0}^{t} A\left(x\left(t, K_{n}\right)-x\left(t, K_{0}\right)\right) d t \| \leq \\
\leq
\end{array} \quad\right\| x\left(0, K_{n}\right)-x\left(0, K_{0}\right)\|+\| A\left\|\int_{0}^{t}\right\| x\left(t, K_{n}\right)-x\left(t, K_{0}\right) \| d t+ \\
& +\left\|\int_{0}^{t} \int_{0}^{t} K_{n}\left(t_{1}-s\right) x\left(s, K_{n}\right) d s d t_{1}-\int_{0}^{t} \int_{0}^{t} K_{n}\left(t_{1}-s\right) x\left(s, K_{0}\right) d s d t_{1}\right\|+ \\
& \quad+\left\|\int_{0}^{t} \int_{0}^{t} K_{n}\left(t_{1}-s\right) x\left(s, K_{0}\right) d s d t_{1}-\int_{0}^{t} \int_{0}^{t} K_{0}\left(t_{1}-s\right) x\left(s, K_{0}\right) d s d t_{1}\right\| \leq \\
& \quad \leq\left\|x\left(0, K_{n}\right)-x\left(0, K_{0}\right)\right\|+\|A\| \int_{0}^{t}\left\|x\left(t, K_{n}\right)-x\left(t, K_{0}\right)\right\| d t+ \\
& \quad+\left\|\int_{0}^{t} \int_{0}^{t} K_{n}\left(t_{1}-s\right)\left[x\left(s, K_{n}\right)-x\left(s, K_{0}\right)\right] d s d t_{1}\right\|+ \\
& \quad+\left\|\int_{0}^{t} \int_{0}^{t}\left[K_{n}\left(t_{1}-s\right)-K_{0}\left(t_{1}-s\right)\right] x\left(s, K_{0}\right) d s d t_{1}\right\|= \\
& =\left\|x\left(0, K_{n}\right)-x\left(0, K_{0}\right)\right\|+\|A\| \int_{0}^{t}\left\|x\left(t, K_{n}\right)-x\left(t, K_{0}\right)\right\| d t+ \\
& \quad+\left\|\int_{0}^{t} \int_{s}^{t} K_{n}\left(t_{1}-s\right) d t_{1}\left[x\left(s, K_{n}\right)-x\left(s, K_{0}\right)\right] d s\right\|+ \\
& \quad+\left\|\int_{0}^{t} \int_{s}^{t}\left[K_{n}\left(t_{1}-s\right)-K_{0}\left(t_{1}-s\right)\right] d t_{1} x\left(s, K_{0}\right) d s\right\|
\end{aligned}
$$

Further we use the following estimates

(a)

$$
\begin{aligned}
& \left\|\int_{0}^{t} \int_{s}^{t} K_{n}\left(t_{1}-s\right) d t_{1}\left[x\left(s, K_{n}\right)-x\left(s, K_{0}\right)\right] d s\right\| \leq \\
& \quad \leq\left\|\int_{0}^{t} \int_{s}^{t}\left|K_{n}\left(t_{1}-s\right)\right| d t_{1}\left[x\left(s, K_{n}\right)-x\left(s, K_{0}\right)\right] d s\right\| \leq \\
& \quad \leq\left\|\int_{0}^{T}\left|K_{n}(\tau)\right| d \tau\right\| \int_{0}^{t}\left\|x\left(s, K_{n}\right)-x\left(s, K_{0}\right)\right\| d s
\end{aligned}
$$

Due to Cauchy-Bunyakovsky inequality

$$
\left\|\int_{0}^{T}\left|K_{n}(\tau)\right| d \tau\right\| \leq T \int_{0}^{T}\left\|K_{n}(\tau)\right\|^{2} d \tau=T M
$$


where $\int_{0}^{T}\left\|K_{n}(\tau)\right\|^{2} d \tau=M<\infty$ due to condition (3.2), we can continue (3.4) as

$$
\begin{aligned}
& \left\|\int_{0}^{t} \int_{s}^{t} K_{n}\left(t_{1}-s\right) d t_{1}\left[x\left(s, K_{n}\right)-x\left(s, K_{0}\right)\right] d s\right\| \leq \\
& \quad \leq T M \int_{0}^{t}\left\|x\left(s, K_{n}\right)-x\left(s, K_{0}\right)\right\| d s
\end{aligned}
$$

(b)

$$
\begin{aligned}
& \left\|\int_{0}^{t} \int_{s}^{t}\left[K_{n}\left(t_{1}-s\right)-K_{0}\left(t_{1}-s\right)\right] d t_{1} x\left(s, K_{0}\right) d s\right\| \leq \\
& \quad \leq \int_{0}^{t}\left\|\int_{s}^{t}\left[K_{n}\left(t_{1}-s\right)-K_{0}\left(t_{1}-s\right)\right] d t_{1}\right\|\left\|x\left(s, K_{0}\right)\right\| d s \leq \\
& \quad \leq \int_{0}^{t} \int_{s}^{t}\left\|K_{n}\left(t_{1}-s\right)-K_{0}\left(t_{1}-s\right)\right\|^{2} d t_{1}\left(t_{1}-s\right)\left\|x\left(s, K_{0}\right)\right\| d s \leq \\
& \quad \leq \int_{0}^{T} \int_{0}^{T}\left\|K_{n}\left(t_{1}-s\right)-K_{0}\left(t_{1}-s\right)\right\|^{2} d t_{1} T\left\|x\left(s, K_{0}\right)\right\| d s
\end{aligned}
$$

Applying (3.6) and (3.7) in (3.3) we get

$$
\begin{aligned}
& \left\|x\left(t, K_{n}\right)-x\left(t, K_{0}\right)\right\| \leq \\
& \quad \leq\left\|x\left(0, K_{n}\right)-x\left(0, K_{0}\right)\right\|+\|A\| \int_{0}^{t}\left\|x\left(t, K_{n}\right)-x\left(t, K_{0}\right)\right\| d t+ \\
& \quad+T M \int_{0}^{t}\left\|x\left(s, K_{n}\right)-x\left(s, K_{0}\right)\right\| d s+ \\
& \quad+\int_{0}^{T} \int_{0}^{T}\left\|K_{n}\left(t_{1}-s\right)-K_{0}\left(t_{1}-s\right)\right\|^{2} d t_{1} T\left\|x\left(s, K_{0}\right)\right\| d s
\end{aligned}
$$

Using in (3.8) Gronwall lemma we get

$$
\begin{aligned}
& \left\|x\left(t, K_{n}\right)-x\left(t, K_{0}\right)\right\| \leq \\
& \quad \leq\left(\left\|x\left(0, K_{n}\right)-x\left(0, K_{0}\right)\right\|+\right. \\
& \left.\quad+T \int_{0}^{T} \int_{0}^{T}\left\|K_{n}\left(t_{1}-s\right)-K_{0}\left(t_{1}-s\right)\right\|^{2} d t_{1}\left\|x\left(s, K_{0}\right)\right\| d s\right) e^{(\|A\|+T M) t}
\end{aligned}
$$

By virtue to (3.2) right side of (3.9) tends to zero as $n \rightarrow \infty$. 


\section{ESTIMATION PROBLEMS IN HILbERT SPACE}

Consider identification problem of system

$$
\begin{gathered}
\frac{d x}{d t}=f(t, x, \alpha)+\int_{t_{0}}^{t} g(t, s, x(s), \beta) d s \\
x\left(t_{0}\right)=x_{0}
\end{gathered}
$$

where $x(t) \in \mathbb{R}^{n}, \alpha \in \mathbb{W}_{1}, \beta \in \mathbb{H}_{2}$. Here $\mathbb{W}_{1}, \mathbb{W}_{2}$ are abstract Hilbert spaces. Introduce denotions

$$
f_{1}(t, x, \theta)=f(t, x, \alpha)+\int_{t_{0}}^{t} g(t, s, x(s), \beta) d s, \quad \theta=\left(\begin{array}{c}
\alpha \\
\beta
\end{array}\right) .
$$

Assume that values of parameters $\alpha$ and $\beta$ are unknown and their aprioric sets ( $G_{\alpha}$ and $G_{\beta}$ respectively) are only given. Consider the following problems.

Problem 1. Knowing values of function $x(s)$ and $\frac{d x(s)}{d s}, s \leq T$ with some unknown error, find estimates of parameters $\alpha$ and $\beta$.

Problem 2. For given function $y(t)$ such that

$$
y(t)=h(t, x(t))+\eta(t), t_{0} \leq t \leq T, \text { where } y \in \mathbb{R}^{m},
$$

$h$ is known vector-valued function, $\eta(t)$ is some unknown function belonging to set $G_{\eta}$ from space $L_{2}\left(t_{0}, T\right)$ find estimates of parameters $\alpha$ and $\beta$, and estimate of function $x(s), s \geq T$.

Firstly note that these problems are covered by problems of estimation of differential equation solutions in Hilbert spaces.

Indeed if we introduce function $\theta(t)$ with values in $\mathbb{H}=\mathbb{H}_{1} \times \mathbb{H}_{2}$ as solution of equation

then we get system of equations

$$
\frac{d \theta}{d t}=0, \theta\left(t_{0}\right)=\theta
$$

$$
\begin{aligned}
& \frac{d x(t)}{d t}=f_{1}(s, x(s), \theta(s)), \\
& x\left(t_{0}\right)=x_{0}, \\
& \frac{d \theta}{d t}=0, \theta\left(t_{0}\right)=\theta .
\end{aligned}
$$

Thus in case of Problem 1 we should find an estimate $\theta(t)$ as solution of differential equation observing $x(t), t_{0}<t<T$ satisfying equation (4.1).

In case of the Problem 2 the problem of estimation of $\theta$ and $x(s)$ is reduced to estimate of differential equation solution in Hilbert space $\mathbb{R}^{n} \times \mathbb{W}$. 
Hence it is reasonable to investigate problems on estimation of differential equation solution parameters in Hilbert space firstly.

Firstly consider case of linear differential equation on $\mathbb{W}$

$$
\begin{array}{r}
\frac{d x}{d t}=\mathcal{A}(t) x(t)+\mathscr{B}(t) f_{1}(t) \\
x\left(t_{0}\right)=x_{0},
\end{array}
$$

where $\mathcal{A}(t) \in \mathfrak{L}(\mathbb{H}, \mathbb{W}), f_{1}(t)$ is unknown function from $L_{2}\left(\left(t_{0}, T\right), \mathbb{F}_{1}\right)$, where $\mathbb{F}_{1}$ is some Hilbert space, $\mathscr{B}(t) \in \mathfrak{L}\left(\mathbb{F}_{1}, \mathbb{W}\right)$, norms $\|\mathcal{A}(t)\|$ and $\|\mathcal{B}(t)\|$ are continuous functions, $x_{0}$ is unknown vector from $\mathbb{H}$.

Note we mean solution of the following integral equation as generalized solution of equation (4.4)

$$
x(t)=\int_{t_{0}}^{t} \mathscr{A}(s) x(s) d s+\int_{t_{0}}^{t} \mathscr{B}(s) f_{1}(s) d s
$$

Such solution exists and is unique (it was proved in [16]). Besides that, let observations are given

$$
y(t)=\mathscr{H}(t, y(\bullet), x(\bullet))+\mathscr{D}(t) f_{2}(t)
$$

where $\mathbb{F}_{2}, Y$ are Hilbert spaces, $\|\mathscr{D}(t)\|$ is continuous with respect to $t, f_{2}$ is unknown function from space $\mathbb{F}_{2}, \mathscr{H}(t, y(\bullet), x(\bullet))$ depends on observations $y(s), s<t$ and at fixed $t$ and $y$ is mapping the space $\mathbb{W}$ into $\mathrm{Y}$.

Also let triple $\left(x_{0}, f_{1}(\bullet), f_{2}(\bullet)\right)$ belong to some set $G$ of Hilbert space $\mathbb{R} \times$ $L_{2}\left(\left(t_{0}, T\right), \mathbb{F}_{1}\right) \times L_{2}\left(\left(t_{0}, T\right), \mathbb{F}_{2}\right)$.

Definition 2. Aposterioric estimate of vector $\delta x(T)$, where $\delta \in \mathfrak{L}\left(\mathbb{M}, \mathbb{F}_{3}\right), \mathbb{F}_{3}$ is Hilbert space is vector $\delta \hat{x}(T)$, where $\hat{x}(T)$ is solution of equation

$$
\begin{gathered}
\frac{d \hat{x}}{d t}=\mathcal{A}(t) \hat{x}(t)+\mathscr{B}(t) \hat{f}_{1}(t), \\
\hat{x}\left(t_{0}\right)=\hat{x}_{0},
\end{gathered}
$$

and pair $\left(\hat{x}_{0}, \hat{f}_{1}\right)$ belongs to aposterioric set $G_{y}$ determined by the following way

$$
G_{y}=\left\{\left(x_{0}, f_{1}\right):\left(x_{0}, f_{1}, f_{2}\right) \in G_{y}^{(1)}\right\},
$$

where

$$
G_{y}^{(1)}=\left\{\left(x_{0}, f_{1}, f_{2}\right):\left(x_{0}, f_{1}, f_{2}\right) \in G, y(t)=\mathscr{H}(t, y(\bullet), x(\bullet))+\mathscr{D}(t) f_{2}(t), t_{0} \leq t \leq T\right\}
$$

It is obvious that if $\mathscr{H}(t, y(\bullet), x(\bullet))$ is continuous mapping with respect to $x$ and set $G$ is bounded then aposterioric set is also bounded. 
Remark 1 . If $\hat{x}_{0}, \hat{f}_{1}$ are aposteriric estimates of vectors $x_{0}$ and $f_{1}$ correspondingly then inequality holds

$$
\left\{\left\|\hat{x}_{0}-x_{0}\right\|^{2}+\left\|\hat{f}_{1}-f_{1}\right\|^{2}\right\}^{1 / 2} \leq \sup _{\left(x_{0}, f_{1}\right) \in G_{y}}\left[\left\|\hat{x}_{0}-x_{0}\right\|^{2}+\left\|\hat{f}_{1}-f_{1}\right\|^{2}\right]^{1 / 2}=\sigma_{a}^{(1)}
$$

On the other hand for minimax aposterioric estimates $\hat{\hat{x}} x_{0}, \hat{{ }^{\prime}} f_{1}$ determined by equality

$$
\begin{gathered}
\inf _{\left(\hat{x}_{0}, \hat{f}_{1}\right) \in G_{y}\left(x_{0}, f_{1}\right) \in G_{y}}\left\{\left\|\hat{x}_{0}-x_{0}\right\|^{2}+\left\|\hat{f}_{1}-f_{1}\right\|^{2}\right\}^{1 / 2} \leq \\
\leq \sup _{\left(x_{0}, f_{1}\right) \in G_{y}}\left[\left\|\hat{x_{0}}-x_{0}\right\|^{2}+\left\|\hat{} f_{1}-f_{1}\right\|^{2}\right]^{1 / 2}=\sigma_{a}
\end{gathered}
$$

the following ineqality holds

$$
\sigma_{a} \leq \sigma_{a}^{(1)}
$$

Remark 2. Let $G$ be bounded closed set. Then

$\sup _{\left(x_{0}, f_{1}\right) \in G_{y}}\left[\left\|\hat{x}_{0}-x_{0}\right\|^{2}+\left\|\hat{f}_{1}-f_{1}\right\|^{2}\right]^{1 / 2}=\sup _{\left(l_{1}, l_{1}\right)+\left(l_{2}, l_{2}\right) \leq 1}\left[\sigma_{l}+|L(\hat{f})-\hat{L}(f)|\right]^{1 / 2}$

where

$$
\begin{gathered}
\sigma_{l}=\frac{1}{2}\left[\sup _{G_{y}} L(f)-\inf _{G_{y}} L(f)\right] \\
\hat{L}(f)=\frac{1}{2}\left[\sup _{G_{y}} L(f)+\inf _{G_{y}} L(f)\right] \\
L(f)=\left(l_{1}, x_{0}\right)+\left(l_{2}, f_{1}\right) \\
L(\hat{f})=\left(l_{1}, \hat{x}_{0}\right)+\left(l_{2}, \hat{f}_{1}\right)
\end{gathered}
$$

Proof. Since

$$
\left\|\hat{x}_{0}-x_{0}\right\|^{2}+\left\|\hat{f}_{1}-f_{1}\right\|^{2}=\sup _{\left(l_{1}, l_{1}\right)+\left(l_{2}, l_{2}\right) \leq 1}[L(\hat{f})-L(f)]^{2}
$$

then

$$
\sigma_{a}^{(1)}=\sup _{\left(l_{1}, l_{1}\right)+\left(l_{2}, l_{2}\right) \leq 1} \sup _{f \in G_{y}}|L(\hat{f})-L(f)|
$$

Note that $L(f)$ is changing within

$$
\inf _{G_{y}} L(f) \leq L(f) \leq \sup _{G_{y}} L(f)
$$

or

$$
|L(f)-\hat{L}(f)| \leq \sigma_{l}
$$


Remark 3. Let set $G_{y}$ be bounded and closed, and also centrally symmetric with respect to vector $\bar{x}_{0}, \bar{f}_{1}$ (that is if $\left(x_{0}-\bar{x}_{0}, f_{1}-\bar{f}_{1}\right) \in G_{y}$ then $-\left(x_{0}-\bar{x}_{0}, f_{1}-\bar{f}_{1}\right) \in$ $G_{y}$ also). Then it is easy seen that

$$
\begin{gathered}
\sup _{G_{y}} L(f)=\sup _{\tilde{G}_{y}} L(f)+L(\bar{f}) \\
\inf _{G_{y}} L(f)=-\sup _{\tilde{G}_{y}} L(f)+L(\bar{f})
\end{gathered}
$$

where $\tilde{G}_{y}=G_{y}-\left(\bar{x}_{0}, \bar{f}_{0}\right)$ and is centrally symmetric with respect to zero and it implies

$\sigma_{a}^{(1)}=\sup _{\left(l_{1}, l_{1}\right)+\left(l_{2}, l_{2}\right) \leq 1}\left[\sup _{\tilde{G}_{y}} L(f)+|L(\hat{f})-L(\bar{f})|\right] \geq \sup _{\tilde{G}_{y}}\left[\left(x_{0}, x_{0}\right)+\left(f_{1}, f_{1}\right)\right]^{1 / 2}$

Equality sign is reached as $\hat{f}=\bar{f}$. That is $\bar{f}$ is minimax aposterioric estimate with estimation error

$$
\sigma_{a}=\sup _{\tilde{G}_{y}}\left[\left(x_{0}, x_{0}\right)+\left(f_{1}, f_{1}\right)\right]^{1 / 2}
$$

Further we consider case when $\mathscr{H}(t, y(\bullet), x(\bullet))$ depends on $x(\bullet)$ linearly. Moreover,

$$
\mathscr{H}(t, y(\bullet), x(\bullet))=\mathscr{H}(t, y(\bullet)) x(t)
$$

where $\mathscr{H}(t, y(\bullet)) \in \mathfrak{L}(H, Y)$.

Introduce functional

$$
\begin{aligned}
& \mathcal{g}\left(x_{0}, f_{1}, f_{2}\right)=\left(\mathcal{Q}_{0} x_{0}, x_{0}\right)+\int_{t_{0}}^{T}\left(\mathcal{Q}_{1} f_{1}, f_{1}\right) d t+\int_{t_{0}}^{T}\left(\mathcal{Q}_{2} f_{2}, f_{2}\right) d t+ \\
& +\int_{t_{0}}^{T}\left(\lambda(t), y(t)-\mathscr{H}(t, y(\bullet)) x(t)-\mathscr{D}(t) f_{2}(t)\right) d t
\end{aligned}
$$

and consider the following aposterioric set

$$
G_{y}=\left\{\left(x_{0}, f_{1}\right): \mathcal{g}\left(x_{0}, f_{1}, f_{2}\right) \leq 1\right\}
$$

Lemma 3. Aposterioric estimates of the problem (4.4)-(4.6) can be found as a result of solution of the following system of equations with respect to $p(t), \hat{x}(t), \hat{\lambda}(t)$

$$
\begin{gathered}
\left\{\begin{array}{l}
-p^{\prime}(t)-\mathcal{A}^{*}(t) p(t)=\mathscr{H}^{*}(t, y(\bullet)) \hat{\lambda}(t), \\
p(T)=0,
\end{array}\right. \\
\left\{\begin{array}{l}
\frac{d \hat{x}(t)}{d t}=\mathscr{A}(t) \hat{x}(t)+\mathscr{B}(t) \mathcal{Q}_{1}^{-1} \mathscr{B}^{*}(t) p(t), \\
\hat{x}\left(t_{0}\right)=\mathcal{Q}_{0}^{-1} p\left(t_{0}\right),
\end{array}\right. \\
y(t)=\mathscr{H}^{*}(t, y(\bullet)) \hat{x}(t)+\frac{1}{2} \mathscr{D}(t) \mathcal{Q}_{2}^{-1} \mathscr{D}^{*}(t) \hat{\lambda}(t)
\end{gathered}
$$


and have the following form

$$
\hat{f}_{1}(t)=Q_{1}^{-1} \mathscr{B}^{*}(t) p(t), \hat{f}_{2}(t)=\frac{1}{2} Q_{2}^{-1} \mathscr{D}^{*}(t) \hat{\lambda}(t)
$$

Proof. Calculate

$$
\begin{aligned}
& \left.\frac{d}{d \tau} \mathcal{H}\left(x_{0}+\tau w, f_{1}+\tau g_{1}, f_{2}+\tau g_{2}\right)\right|_{\tau=0}=2\left(Q_{0} x_{0}, w\right)+2 \int_{t_{0}}^{T}\left(\mathcal{Q}_{1} f_{1}, g_{1}\right) d t+ \\
& +2 \int_{t_{0}}^{T}\left(Q_{2} f_{2}, g_{2}\right) d t-\int_{t_{0}}^{T}\left(\lambda(t), \mathscr{H}(t, y(\bullet)) x\left(g_{1}\right)-\mathscr{D}(t) g_{2}(t)\right) d t= \\
& =2\left(\mathcal{Q}_{0} x_{0}, w\right)+2 \int_{t_{0}}^{T}\left(\mathcal{Q}_{1} f_{1}, g_{1}\right) d t+2 \int_{t_{0}}^{T}\left(Q_{2} f_{2}, g_{2}\right) d t-\int_{t_{0}}^{T}\left(\mathscr{D}^{*}(t) \lambda(t), g_{2}\right) d t- \\
& -\int_{t_{0}}^{T}\left(\lambda(t), \mathscr{H}(t, y(\bullet)) x\left(g_{1}\right)\right) d t
\end{aligned}
$$

Introduce adjoined function $p(t)$ as solution of equation

$$
-p^{\prime}(t)-\mathscr{A}^{*}(t) p(t)=\mathscr{H}^{*}(t, y(\bullet)) \hat{\lambda}(t)
$$

Hence

$$
\begin{aligned}
& \left.\frac{d}{d \tau} \mathcal{A}\left(x_{0}+\tau w, f_{1}+\tau g_{1}, f_{2}+\tau g_{2}\right)\right|_{\tau=0}=2\left(\mathcal{Q}_{0} x_{0}, w\right)+2 \int_{t_{0}}^{T}\left(\mathcal{Q}_{1} f_{1}, g_{1}\right) d t+ \\
& 2 \int_{t_{0}}^{T}\left(\mathcal{Q}_{2} f_{2}, g_{2}\right) d t-\int_{t_{0}}^{T}\left(\mathcal{D}^{*}(t) \lambda(t), g_{2}\right) d t+(p(T), x(T))-\left(p\left(t_{0}\right), w\right)- \\
& -\int_{t_{0}}^{T}(p(t), \mathcal{A}(t) x(t)) d t-\int_{t_{0}}^{T}\left(p(t), \mathcal{B}(t) g_{1}(t)\right) d t+\int_{t_{0}}^{T}\left(\mathcal{A}^{*}(t) p(t), x(t)\right) d t=0
\end{aligned}
$$

Lemma 4. Let there exists $\mathscr{G}=\left[\mathscr{D}(t) \mathcal{Q}_{2}^{-1} \mathscr{D}^{*}(t)\right]^{-1}$. Then solution of system (4.7)-(4.9) can be found in form

$$
\hat{x}(t)=\mathcal{P}(t) p(t)+q(t)
$$

where $q(t) \in \mathbb{W}$ is solution of problem

$$
\left\{\begin{array}{l}
\frac{d q(t)}{d t}=\mathscr{A}(t) q(t)+2 \mathcal{P}(t) \mathscr{H}^{*}(t, y(\bullet)) \mathcal{E}[y(t)-\mathscr{H}(t, y(\bullet)) q(t)] \\
q\left(t_{0}\right)=\mathcal{O}, \quad \mathcal{O} \text { is null-vector, }
\end{array}\right.
$$

operator $\mathcal{P}(t) \in \mathfrak{L}(\mathbb{H}, \mathbb{H})$ is solution of the following Riccati equation

$$
\left\{\begin{aligned}
\frac{d \mathcal{P}(t)}{d t}= & \mathcal{A}(t) \mathcal{P}(t)+\mathcal{P}(t) \mathcal{A}^{*}(t)-2 \mathcal{P}(t) \mathscr{H}^{*}(t, y(\bullet)) \mathcal{E} \mathcal{H}(t, y(\bullet)) \mathcal{P}(t)+ \\
& +\mathbb{B}(t) \mathcal{Q}_{1}^{-1} \mathscr{B} *(t) \\
\mathcal{P}\left(t_{0}\right)= & \mathcal{Q}_{0}^{-1} .
\end{aligned}\right.
$$


Proof. From (4.11) it follows that

$$
\frac{d \hat{x}(t)}{d t}=\frac{d \mathcal{P}(t)}{d t} p(t)+\mathcal{P}(t) p^{\prime}(t)+q^{\prime}(t)
$$

Using in (4.14) equations (4.7), (4.8) we have

$$
\begin{aligned}
& \mathcal{A}(t) \hat{x}(t)+\mathscr{B}(t) \mathcal{Q}_{1}^{-1} \mathscr{B}^{*}(t) p(t)=\frac{d \mathcal{P}(t)}{d t} p(t)+ \\
& +\mathcal{P}(t)\left[-\mathcal{A}^{*}(t) p(t)-\mathscr{H}^{*}(t, y(\bullet)) \hat{\lambda}(t)\right]+q^{\prime}(t)
\end{aligned}
$$

Due to (4.9) we have

$$
\hat{\lambda}=2 \mathscr{E}[y(t)-\mathscr{H}(t, y(\bullet)) \hat{x}(t)]
$$

Since (4.15) it follows that

$$
\begin{aligned}
\{ & \mathcal{A}(t) \mathcal{P}(t)+B(t) Q_{1}^{-1} \mathcal{B}^{*}(t)-\frac{d \mathcal{P}(t)}{d t}+ \\
& \left.+\mathcal{P}(t) A^{*}(t)-2 \mathcal{P}(t) \mathscr{H}^{*}(t, y(\bullet)) \mathcal{E H}(t, y(\bullet)) \mathcal{P}(t)\right\}= \\
= & \left\{-\mathcal{A}(t) q(t)-2 \mathcal{P}(t) \mathscr{H}^{*}(t, y(\bullet)) \mathcal{E} y(t)+\right. \\
& \left.+2 \mathcal{P}(t) \mathscr{H}^{*}(t, y(\bullet)) \mathcal{E} \mathscr{H}(t, y(\bullet)) q(t)+q^{\prime}(t)\right\}
\end{aligned}
$$

Thus we obtained problem (4.12) for $q(t)$ and (4.13) for $\mathcal{P}(t)$.

At last we consider case of differential equation (4.4) over space $\mathbb{R}^{n} \times \mathbb{W}$, where $\mathbb{W}$ is abstract Hilbert space and operator $\mathscr{H}(t, y(\bullet))$ has the form $\mathscr{H}(t, y(\bullet))=$ $\left(H_{1}(t), \mathcal{O}\right)$, where $H_{1}(t) \in \mathbb{R}^{m \times n}, \mathcal{O}$ is null-vector. Let vector $x(t) \in \mathbb{R}^{n} \times \mathbb{U}$ has presentation $x=\left(\begin{array}{c}x_{1} \\ x_{2}\end{array}\right)$, where $x_{1} \in \mathbb{R}^{n}, x_{2} \in \mathbb{R}$. Let we consider space $\mathbb{R}^{m}$ as space of observations $Y$ and $Q_{2} \in \mathbb{R}^{m \times m}$. Then

$$
y(t)=H_{1}(t) x_{1}(t)+\mathscr{D}(t) f_{2}(t),
$$

where $\mathscr{D}(t) f_{2}(t) \in \mathbb{R}^{m}$.

Further applying result [4] on presentation of linear operator $A \in \mathfrak{L}\left(\mathbb{R}^{n} \times \mathbb{U}, \mathbb{R}^{n} \times \mathbb{U}\right)$, where $\mathbb{W}$ is abstract Hilbert space in the form

$$
\mathcal{A}=\left(\begin{array}{ll}
\mathcal{A}_{11} & \mathcal{A}_{12} \\
\mathcal{A}_{21} & \mathcal{A}_{22}
\end{array}\right),
$$

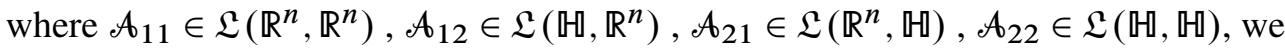
lead to the following presentations of operators

$$
\begin{aligned}
& \mathcal{A}(t)=\left(\begin{array}{ll}
\mathcal{A}_{11}(t) & \mathcal{A}_{12}(t) \\
\mathcal{A}_{21}(t) & \mathcal{A}_{22}(t)
\end{array}\right), \\
& \mathcal{P}(t)=\left(\begin{array}{ll}
\mathcal{P}_{11}(t) & \mathcal{P}_{21}(t) \\
\mathcal{P}_{21}(t) & \mathcal{P}_{22}(t)
\end{array}\right),
\end{aligned}
$$




$$
\begin{gathered}
\mathcal{Q}_{1}^{-1}=\left(\begin{array}{ll}
\mathcal{Q}_{1}^{11} & \mathcal{Q}_{1}^{12} \\
\mathcal{Q}_{1}^{21} & \mathcal{Q}_{1}^{22}
\end{array}\right), \\
\mathcal{B}(t)=\left(\begin{array}{ll}
\mathcal{B}_{11}(t) & \mathcal{B}_{12}(t) \\
\mathcal{B}_{21}(t) & \mathcal{B}_{22}(t)
\end{array}\right),
\end{gathered}
$$

where elements of operators belong to corresponding spaces.

In this case the problem (4.12) for $q(t)=\left(\begin{array}{l}q_{1}(t) \\ q_{2}(t)\end{array}\right)$ results in problems

$\left\{\begin{array}{l}\frac{d q_{1}(t)}{d t}=\mathscr{A}_{11}(t) q_{1}(t)+\mathcal{A}_{12}(t) q_{2}(t)+2 \mathcal{P}_{11}(t) H_{1}^{*}(t) \mathscr{E}\left[y(t)-H_{1}(t) q_{1}(t)\right] \\ q_{1}\left(t_{0}\right)=0,\end{array}\right.$

$\left\{\begin{array}{l}\frac{d q_{2}(t)}{d t}=\mathcal{A}_{21}(t) q_{1}(t)+\mathcal{A}_{22}(t) q_{2}(t)+2 \mathcal{P}_{21}(t) H_{1}^{*}(t) \mathcal{E}\left[y(t)-H_{1}(t) q_{1}(t)\right] \\ q_{2}\left(t_{0}\right)=\mathcal{O}\end{array}\right.$ and four problems for components of operator $\mathcal{P}(t)$.

\section{EXAMPLE}

Let along interval of time $\left(0, t_{1}\right)$ there are observed the function $x(t) \in \mathbb{R}^{1}$ and its derivative $\frac{d x(t)}{d t}$ with error $v(t)$. The function $x(t)$ is solution of the equation

$$
\frac{d x(t)}{d t}=a(t) x(t)+\int_{0}^{1} \theta(t) x(t-\tau) d \tau .
$$

Function $a(t)$ is known and is continuous on $\left[0, t_{1}\right], \theta(t)$ is unknown function from space $L_{2}(0,1)$.

Assume, there are known restrictions for $\theta(t)$ and measurements errors $v(t)$. We determine the following set in the form

$$
G=\left\{(\theta, v): q_{0}^{2} \int_{0}^{1} \theta^{2}(t) d t+q_{2}^{2} \int_{0}^{t_{1}} v^{2}(t) d t \leq \gamma^{2}\right\}
$$

Then aposterioric set will have the form

$$
G_{y}=\left\{\theta: \mathscr{g}_{y} \leq \gamma^{2}\right\},
$$

where $\mathcal{g}_{y}(\theta)=q_{0}^{2} \int_{0}^{1} \theta^{2}(\tau) d \tau+\int_{0}^{t_{1}}\left(y(t)-\int_{0}^{1} \theta(\tau) x(\tau-s) d s\right)^{2} q_{2}^{2}$, where $y(t)=$ $\frac{d x(t)}{d t}-a(t) x(t)$.

Rewrite the problem in vector-like form. The observations are

$$
y(t)=(h(t), \theta)_{L_{2}(0,1)}+v(t)
$$

Hereinafter $(h(t), \theta)=(h(t), \theta)_{L_{2}(0,1)}:=\int_{0}^{1} \theta(\tau) x(t-\tau) d \tau$.

We write the equation for the parameter $\theta$

$$
\frac{d \theta}{d t}=0, \quad \theta(0)=\theta
$$


For aposterioric estimate of the parameter $\theta(t)=\theta$ which is based on observations till the instant $t_{1}$ we get the following boundary value problem

$$
\begin{aligned}
-\frac{d \hat{p}}{d t} & =h(t) q_{2}^{2}(y(t)-(h(t), \hat{\theta}(t))), \\
\hat{p}\left(t_{1}\right) & =0, \\
\frac{d \hat{\theta}(t)}{d t} & =0, \\
\hat{\theta}(0) & =q_{0}^{-2} \hat{p}(0)
\end{aligned}
$$

Due to these system of equations we can get integral equation for determining the estimate of the function $\hat{\theta}(\tau)$

$$
\hat{\theta}(\tau)+\int_{0}^{1} R\left(\tau, \tau_{1}\right) \hat{\theta}\left(\tau_{1}\right) d \tau_{1}=\int_{0}^{1} x(t-\tau) q_{0}^{-2} q_{2}^{2} y(t) d t,
$$

where $R\left(\tau, \tau_{1}\right)=\int_{0}^{t_{1}} x(t-\tau) x\left(t-\tau_{1}\right) d t q_{0}^{-2} q_{2}^{2}$.

Based on the results which were obtained above it follows that the estimate of parameter $\theta\left(t_{1}\right)$ using the observations till the instant $t_{1}$ satisfies to the differential equation

$$
\begin{aligned}
\frac{d \hat{\theta}(t)}{d t} & =\mathcal{P}^{2}(t) q_{2}^{2} h(t)(y(t)-(h(t), \hat{\theta}(t))), \\
\hat{\theta}(0) & =0 .
\end{aligned}
$$

Here operator-function $\mathcal{P}(t)$ is solution of the equation

$$
\begin{aligned}
\frac{d \mathcal{P}(t)}{d t} & =-\mathcal{P}(t) h(t) \otimes h(t) q_{2}^{2} \mathcal{P}(t), \\
\mathcal{P}(0) & =q_{0}^{-2} \mathcal{E},
\end{aligned}
$$

which has the solution

$$
\mathcal{P}(t)=\left(q_{0}^{-2} \mathscr{E}+\int_{0}^{t} h(t) \otimes h(\tau) d \tau q_{2}^{2}\right)^{-1},
$$

where $h(\tau) \otimes h(\tau)$ is linear continuous operator mapping in the following way

$$
h(\tau) \otimes h(\tau) x=h(\tau)(h(\tau), x)
$$

Aposterioric error of such estimate has the form

$$
\sigma_{a}=\sup _{\theta \in G_{y}}\left|\hat{\theta}\left(t_{1}\right)-\theta\right|=\left(\gamma^{2}-\mathscr{\not}_{y}(\hat{\theta})\right)^{1 / 2}\left(q_{0}^{-2}+q_{2}^{2} \lambda_{\min }(\mathscr{H})\right)^{-1 / 2},
$$

where $\lambda_{\min }(\mathscr{H})$ is minimal eigenvalue of operator $\mathscr{H}$, where $\mathscr{H}:=\int_{0}^{t_{1}} h(\tau) \otimes h(\tau) d \tau$. 
Note that

$$
\sigma_{a} \leq \gamma\left(q_{0}^{-2}+q_{2}^{2} \lambda_{\min }(\mathscr{H})\right)^{-1 / 2}
$$

\section{CONCLusions}

Thus the work considers the problem of differential equation parameter identification given in Hilbert space. Conditions of existence of problem solutions are established. They correspond to conditions of continuous dependence of solutions of differential equations on parameters. In case of linear model and space $L_{2}$ condition means convergence in mean quadratic sense on aprioric set.

A constructive algorithm of identification problem solution in Hilbert space is developed. It results in solution of corresponding boundary value problem. The way of its reduction to Cauchy problems is offered, one partial case allowing problem solution not only in operator form is considered.

In the next works solution of Problems 1 and 2 should be considered in case of models given by integro-differential equations.

\section{ACKNOWLEDGEMENT}

The authors would like to express their gratitude to the reviewers for the valuable comments.

\section{REFERENCES}

[1] "Lower semicontinuous functionals," in Systems \& Control: Foundations \& Applications. Birkhäuser-Verlag, 2005, pp. 205-219. [Online]. Available: https: //doi.org/10.1007/3-7643-7357-1_7. doi: 10.1007/3-7643-7357-1_7

[2] B. Bublik, V. Y. Danilov, and A. Nakonechny, Some problems of observation and control in linear systems. Kiev: UMK PO (in Russian), 1988.

[3] A. Favini and H. Tanabe, "Identification Problems for Integrodifferential Equations with Delay: An Improvement of the Results by G. Di Blasio and A. Lorenzi," Funkcialaj Ekvacioj-Serio Internacia, vol. 60, no. 2, pp. 133-170, AUG 2017, doi: 10.1619/fesi.60.133.

[4] I. Gohberg and M. G. Krein, Theory and applications of Volterra operators in Hilbert space. American Mathematical Soc., 1970, vol. 24.

[5] K. Gopalsamy, Stability and oscillations in delay differential equations of population dynamics. Springer Science \& Business Media, 2013, vol. 74.

[6] J. K. Hale and S. M. V. Lunel, Introduction to functional differential equations. Springer Science \& Business Media, 2013, vol. 99.

[7] J.-M. Jeong and S.-H. Cho, "Identification problems of retarded differential systems in hilbert spaces," Evolution Equations and Control Theory, vol. 6, no. 1, pp. 77-91, MAR 2017, doi: 10.3934/eect.2017005.

[8] E. A. Kapustyan and A. G. Nakonechnyi, "The minimax problems of pointwise observation for a parabolic boundary-value problem," Journal of Automation and Information Sciences, vol. 34, no. $5,2002$.

[9] I. M. Lyashenko and A. Mukoyed, Modeling biological and ecological processes. Kyiv: Kyiv University, 2002. 
[10] V. Martsenyuk, "Integro-differentual models with memory in population dynamics problems," Journal of Automation and Information Sciences, vol. 36, no. 10, pp. 56-65, 2004, doi: 10.1615/JAutomatInfScien.v36.i10.70.

[11] V. Martsenyuk and O. Nakonechny, "Models and methods of population dynamics for software environment supporting medical system researches," vol. 2007-January, 2007, pp. 195202. [Online]. Available: https://www.scopus.com/inward/record.uri?eid=2-s2.0-84958048021\& partnerID $=40 \& \mathrm{md} 5=\mathrm{c} 5 \mathrm{f} 7 \mathrm{c} 7 \mathrm{f} 04689 \mathrm{f} 85 \mathrm{e} 11 \mathrm{ffac} 94 \mathrm{e} 6 \mathrm{c} 2726 \mathrm{a}$

[12] V. Marzeniuk and A. Nakonechny, System analysis methods of medical and biological processes. Ternopil: Ukrmedknuha, 2003.

[13] A. G. Nakonechny, Minimax estimation of functionals of variational equation solutions in Hilbert spaces. Kiev: KGU (in Russian), 1985.

[14] A. Nakonechny and V. Marzeniuk, "Uncertainties in medical processes control," Lecture Notes in Economics and Mathematical Systems, vol. 581, pp. 185-192, 2006, doi: 10.1007/3-540-352627_11.

[15] O. G. Nakonechnyi, O. Trofymchuk, I. Trofimova, and D. Cherniy, Modeling and analysis of global biospherical processes. Kyiv: Kyiv University, 2002.

[16] F. P. Vasiliev, "Numerical methods for solution of extreme problems," p. 518, 1980.

[17] S. Zhuk and O. Nakonechnii, "Minimax state estimates for abstract neumann problems," Minimax Theory and its Applications, vol. 3, no. 1, pp. 1-21, 2018.

Authors' addresses

O. Nakonechnyi

Taras Shevchenko National University of Kyiv, Department of System Analysis and Decision Making Theory, 4d Glushkov Av., 03680 Kyiv, Ukraine

E-mail address: a.nakonechniy@gmail.com

V. Martsenyuk

University of Bielsko-Biala, Department of Computer Science, 2 Willowa St., 43-309 Bielsko-Biala, Poland

E-mail address: vmart senyuk@ath.bielsko.pl 